\title{
Growth Performance of Eucalypt Clones in Tanzania
}

\author{
Pima N.E. ${ }^{1, *}$, Chamshama S.A.O. ${ }^{2}$, Iddi S. ${ }^{3}$, Maguzu J. ${ }^{1}$ \\ ${ }^{1}$ Tanzania Forestry Research Institute, Tanzania \\ ${ }^{2}$ Department of Forest Biology, Sokoine University of Agriculture, Tanzania \\ ${ }^{3}$ Department of Wood Utilization, Sokoine University of Agriculture, Tanzania
}

Copyright $\subseteq 2016$ by authors, all rights reserved. Authors agree that this article remains permanently open access under the terms of the Creative Commons Attribution License 4.0 International License

\begin{abstract}
A research study was conducted to assess the growth performance of Eucalypt clones in Tanzania. Eucalypt clones of Eucalyptus grandis x E. camaldulensis (GC), E. grandis x E. urophylla (GU), E. grandis x E. tereticornis (GT) were established in Lushoto, Kwamarukanga, Kibaha and Tabora sites. Survival, Diameter at breast height, height and biomass were collected while volume and basal area were evaluated. All assessed variable were subjected to ANOVA. Significant $(p<0.05)$ clonal difference in survival, Dbh, height, basal area, volume and biomass between clones was observed. Survival was $>90 \%$ in Lushoto and Kwamarukanga sites where as the other sites showed intermediate and low survival. Clones at Lushoto site had highest Dbh, height, basal area, volume, Mean annual increment and biomass followed by clones at Kwamarukanga, Kibaha and Tabora sites. It was concluded that at 8 year old Eucalypt clones showed good survival and growth needed for various uses. This study recommended that GC581, GC584 and GU608 are better for Lushoto, GC15, GC167 and GC940 for Kibaha, GC514, GT529 and GC940 for Kwamarukanga and GC15, GC584 and GC940 for Tabora. The outstanding clones are recommended for planting in areas with climatic conditions similar to the sites where they were tested.
\end{abstract}

Keywords Eucalypt Clones, Survival, Growth Performance, Productivity, Tanzania

\section{Introduction}

Eucalyptus is among the most widely cultivated forest trees in the world (over 22 million hectares (ha) [1]. The major Eucalyptus growing countries includes Brazil (3.7 mil ha), India (2.5mil ha) and China (1.7 mil ha). In Africa, South Africa has the largest area under Eucalyptus plantations of about half a million ha [2]. In Tanzania, it is estimated that there are about 25000 ha of Eucalypts plantations [3] of which 4665 ha are grown by government and the rest are grown by the private sector and small-scale farmers [4]. Eucalyptus species that are commonly planted in
Tanzania are E. saligna, E. grandis, E. camaldulensis, E. globules, E. viminalis, E. citriodora, E. regnas, $E$. microtheca, E. tereticornis, E. maidenii. E. maculata, E. paniculata, E. resinifera, E.urophylla and E. robusta.

The rapid decrease in indigenous timber tree species coupled with the need for timber and other wood products, has necessitated Tanzania to embark on commercial plantation forestry development in order to meet the increasing demands. Eucalypt clones have been considered as one of the solutions to meet the need for forest products in the country. Eucalypt clones were introduced from Mondi South Africa to East African countries of Tanzania, Kenya and Uganda during the period of 1997 to 2003. In Tanzania, the clones were introduced in 2003 through Tanzania Forestry Research Institute (TAFORI) in order to test their adaptability in the Tanzanian environment before large scale planting. Experiments started in 2004 using Eucalyptus grandis $\mathrm{x}$ E. camaldulensis (GC) clone, E. grandis $\mathrm{x} E$. urophylla (GU) clone, and E. grandis x E. tereticornis (GT) clones. These clones combine desired traits for two species. E. grandis $x$ E. camaldulensis (GC) combines good growth and drought tolerance. E. grandis $x$ E. urophylla (GU) combines good growth and disease resistance. E. grandis $x E$. tereticornis (GT) combines good growth and rooting ability. The clones are preferred for their fast growth with a short rotation, wide adaptability to site conditions, produce better quality wood and more uniform stands than most indigenous trees [5]. Eucalypt clones are mainly used in many applications in house construction, production of fuel wood, poles, telecommunication posts, fencing posts, electricity transmission poles, pulp and timber [6]. They contribute significantly to reduce the wide gap between demand and production of wood in the shortest possible time [7], thus reducing pressure from the few remaining natural forests [8].

Several studies have reported the significant growth performance of Eucalypt clones in the world [9-10]. The results from these studies shows that Eucalypts hybrid has similar or better growth than their parent and significant differences in growth between hybrid clones may be attributed to genetic constitution as well as environmental factors like soil $\mathrm{pH}$, mean annual rainfall and mean 
temperature. In Tanzania, only a study by [11] reported the growth and survival of 4 year old Eucalypt hybrid clones. The results showed significant survival and growth difference within and between sites. The results also show species site specific performance where GCs and GT survived and performed well in low land areas. However, little research has been done on the growth and survival of these clones at eight year old although there is significant/considerable area planted with Eucalypt clones. Thus, the main objective of this study was to assess the growth performance of Eucalypt hybrid clones in four sites. Therefore, the result from this study provides information on the best clones to be grown in a given agro-ecological zone of Tanzania.

\section{Materials and Methods}

\section{Study area Description}

The study was conducted in four agro-ecological zones of Tanzania namely Highland (Lushoto), Coast (Kibaha), Inland Plateux (Tabora) and Lowland (Kwamarukanga) (Table 1).

\section{Experimental Design}

The experiments were established by TAFORI in 2004 using Eucalypts clonal material from Mondi South Africa. Randomized complete block design with four replications and 12 treatments (Eucalypt clones) was used to set up these experiments at Lushoto, Kibaha and Kwamarukanga sites and 10 treatments at Tabora site. Each clone type was represented once in each block. Each plot comprised 16 trees spaced at $2.5 \times 2.5 \mathrm{~m}$ in a $4 \times 4$ arrangement. The experiments have 2 guard rows planted to avoid edge effect.

\section{Data Collection}

\section{Growth assessment}

Data on survival, Diameter at Breast Height (Dbh), height and biomass were collected at the age of 8 years in 2012. All trees in the plot were measured for Dbh, while 6-12 trees per plot (small, medium and large size) were measured for height. The tally of diameter growth also gave tree survival data. The Dbh measurements were used to calculate the mean plot basal area. Basal area was derived by summing the individual basal areas of trees within a plot and then the plot basal areas were computed by summing basal area of individual trees in a plot. To obtain basal area per ha $\left(\mathrm{m}^{2} \mathrm{ha}^{-1}\right)$, plot basal areas were divided by plot area in ha. Height, Dbh and survival data were first used to determine three best performers in each studied site.

\section{Sampling and Laboratory Procedures}

Three superior Eucalypt clones in terms of survival, Dbh and height (i.e GC 581, GC 584 and GU 608 for Lushoto; GC 15, GC 167 and GC 940 for Kibaha; GC 514, GT 529 and GC 940 for Kwamarukanga and GC 15, GC 584 and GC 940 for Tabora) were selected. Subjective sampling was applied to select thirty trees $(<10 \mathrm{~cm}, 10-20 \mathrm{~cm}$ and $>20 \mathrm{~cm})$ from three clone type at each site for volume and above ground biomass determination. Before felling, trees were measured for Dbh and height and the total length of the tree were measured after felling. Sampled trees were divided into two main parts: aboveground and belowground. The aboveground part was considered as all biomass above a stump height of $15 \mathrm{~cm}$ and it was further divided into sections namely stem, branches including tops (up to a minimum diameter of $2 \mathrm{~cm}$ ) and twigs (with diameter less than $2 \mathrm{~cm}$ ). Stems and branches were trimmed and cross cut into manageable billets ranging from 1 to $1.5 \mathrm{~m}$ in length. Mid diameter and length of each billet were measured for volume determination. Three sample discs from stem and one disc sample from branches (about $2 \mathrm{~cm}$ thick cut from bark to pith) were extracted and weighed. Stem and branch billets were then weighed and the green weight recorded. Twigs were collected into separate bundles and the green weight of each was taken. Leaves were also collected and the green samples were weighed. The total fresh weight of each component was taken in the field using a balance.

Table 1. Study area description

\begin{tabular}{|c|c|c|c|c|}
\hline \multirow{2}{*}{ Site characteristics } & \multicolumn{4}{|c|}{ Sites } \\
\cline { 2 - 5 } & Lushoto & Kwamarukanga & Kibaha & Tabora \\
\hline Latitude & $04^{0} 47^{\prime} 15^{\prime}{ }^{\prime} \mathrm{S}$ & $05^{0} 15^{\prime} 48^{\prime}$, & $06^{0} 42^{\prime} 39 .{ }^{\prime}{ }^{\prime}$ & $04^{0} 82^{\prime} 86^{\prime}{ }^{\prime}$ \\
\hline Longitude & $38^{0} 17^{\prime} 40^{\prime \prime} \mathrm{E}$ & $38^{0} 30^{\prime} 28^{\prime}$, & $38^{0} 52^{\prime} 52^{\prime}{ }^{\prime}$ & $32^{\circ} 62^{\prime} 97^{\prime}{ }^{\prime}$ \\
\hline Altitude (m.a.s.l) & $1393-148$ & 70 & 104 & 1175 \\
\hline Mean annual rainfall $(\mathrm{mm})$ & 1070 & 1000 & 900 & $700-1000$ \\
\hline Mean temperature $\left({ }^{\circ} \mathrm{C}\right)$ & $7-30$ & $19-32$ & $23-35$ & $48-28$ \\
\hline Soil pH & $4.4-4.5$ & $3.8-4.7$ & $4.5-4.9$ & $4.8-6.2$ \\
\hline Soil Organic carbon $(\%)$ & $2.7-3.6$ & $1.8-2.6$ & $0.68-1.7$ & $1.7-2.9$. \\
\hline Soil texture & Sandy & Sandy clay & Sandy & Sandy \\
\hline
\end{tabular}


Stem, branches and twigs samples were oven dried to constant weight at $103 \pm 20 \mathrm{C}$ while leaves were oven dried at $700 \mathrm{C}$ for 48 hours and after that changes in weight were monitored at intervals of 6 hours until there was no change in weight. The wooden blocks from the stem, branch billets and twigs were soaked in water for one week and then measured for green weight using kitchen scale. The volume of each wood block was determined by water displacement method [12]. Biomass was determined using biomass ratios of sample trees and were computed as the ratio of oven dry weight to the green weight for each tree component namely whole tree, stems, branches, twigs and leaves.

\section{Model Development, Selection and Evaluation}

The biomass for each tree component was computed as the product of biomass ratio and total fresh weight. Site specific models for above ground biomass, stem biomass and volume were developed. Models predicting biomass and volume were based on Dbh only, and on a combination of Dbh and height, as independent variables. Numerous model forms have previously been applied when developing biomass models [13-14]. Four model forms for prediction of biomass (dry weight), which have been commonly adopted previously, were tested. Two of the model forms include Dbh only and two include height in addition.

$$
\begin{gathered}
\mathrm{Y}=\beta_{0} \mathrm{dbh}^{\beta 1} \\
\mathrm{Y}=\operatorname{Exp}\left(\beta_{0}+\beta_{1} \ln \left(\mathrm{htx} \mathrm{dbh}^{2}\right)\right. \\
\mathrm{Y}=\beta_{0} \mathrm{dbh}^{\beta 1} \mathrm{ht}^{\beta 2} \\
\mathrm{Y}=\beta_{0}+\beta_{1} \mathrm{dbh}+\beta_{2} \mathrm{dbh}^{2}
\end{gathered}
$$

(Model form 1)

(Model form 2)

(Model form 3)

(Model form 4)

Where

$$
\begin{gathered}
\mathrm{Y}=\text { Biomass }(\mathrm{kg}) \text { or Volume }\left(\mathrm{m}^{3}\right) \\
\text { Dbh }=\text { Diameter at breast height }(\mathrm{cm}) \\
\mathrm{Ht}=\text { tree total height }(\mathrm{m}) \\
\beta_{0} \beta_{1} \text { and } \beta_{2} \text { are regression coefficients. }
\end{gathered}
$$

The best-fit models were selected based on the Akaike Information Criterion (AIC). AIC takes into account the number of parameters in the models and penalizes them accordingly [13]. $\mathrm{R}^{2}$ reported for all tested models were not used as criteria for selecting final models because the tested model forms had different numbers of parameters. With an increase in number of parameters, a model tends to have larger $\mathrm{R}^{2}$ values regardless of their contribution in explaining the variation in the response variable. Models with insignificant parameter estimates were excluded during the selection process irrespective of AIC values. All models were analysed using Non Linear Programming procedure in SAS programme to estimate the model parameters $\left(\beta o, \beta_{1}\right.$, and $\beta 2$ ). The procedure produces the least squares estimates of the parameters of a nonlinear model through an iteration process. Goodness of fit and model comparisons was evaluated using bias percent. Models with lower bias and AIC were selected and used to predict total volume and tree biomass for trees sampled at Lushoto, Kibaha, Kwamarukanga and Tabora sites (Table 2).

Table 2. Final weighted equations for the aboveground biomass and volume model

\begin{tabular}{|c|c|c|c|}
\hline Sites & Biomass model & $\mathbf{R}^{2}$ & AIC \\
\hline Lushoto & $\mathrm{Y}=0.1274 * \mathrm{Dbh}^{\wedge} 2.6110$ & 0.95 & 290.53 \\
\hline Kibaha & $\mathrm{Y}=0.1379 * \mathrm{Dbh}^{\wedge} 2.4369$ & 0.93 & 256.71 \\
\hline Kwamarukanga & $\mathrm{Y}=0.4124 * \mathrm{Dbh}^{\wedge} 2.1128$ & 0.9 & 256.75 \\
\hline Tabora & $\mathrm{Y}=0.7759 * \mathrm{Dbh}^{\wedge} 1.7913$ & 0.9 & 236.84 \\
\hline \multicolumn{4}{|c|}{ Volume model } \\
\hline Lushoto & $\mathrm{Y}=0.000324 * \mathrm{Dbh}^{\wedge} 2.3988$ & 0.96 & 61.03 \\
\hline Kibaha & $\mathrm{Y}=0.000311 * \mathrm{Dbh}^{\wedge} 2.2087$ & 0.96 & 78.4 \\
\hline Kwamarukanga & $\mathrm{Y}=0.000628 * \mathrm{Dbh}^{\wedge} 2.07599$ & 0.90 & 69.66 \\
\hline Tabora & $\mathrm{Y}=0.000811 * \mathrm{Dbh}^{\wedge} 1.8565$ & 0.94 & 87.08 \\
\hline
\end{tabular}

\section{Data Analysis}

For each tree variable namely survival $(\%)$, Dbh $(\mathrm{cm})$, height $(\mathrm{m})$, basal area $\left(\mathrm{m}^{2} \mathrm{ha}^{-1}\right)$, volume $\left(\mathrm{m}^{3} \mathrm{ha}^{-1}\right)$ biomass production $\left(\mathrm{t} \mathrm{ha}^{-1}\right)$ were subjected to analysis of variance using treatment means. Significant clones' means were separated by Duncan's Multiple Range Test. An ordinal ranking scheme was devised to differentiate overall performance for each clone type when significantly different growth was found. Ranking of treatments in six tree parameters namely survival, height, Dbh, basal area, volume and biomass production was used. For each variable which showed significant variation was assigned the best (assigned 1 point) to worse (assigned 12 points) Lushoto, Kibaha and Kwamarukanga sites or 10 for Tabora. Therefore, ranks were added, averaged and the overall score was taken as a basis of the overall clone performance ranking.

\section{Results}

\section{Survival}

The survival of Eucalypt clones differed significantly $(p<0.05)$ between clones within a site (Table 3$)$. The best overall survival of Eucalypt clone at Lushoto site was obtained for GC 10, GC 15, GC 167, GC 785 and GC 796 $(100 \%)$ whilst the poorest was $84 \%$ for GC 514 . For Kwamarukanga, GC 940, GT 529 and GC 514 were the best survivors with survival of $98.44 \%, 97.10 \%$ and $96.98 \%$ respectively compared to the rest of the clones. At Kibaha site, GC 940, GC 15 and GT 529 had highest survival of $76.34 \%, 66.88 \%$ and $62.08 \%$ respectively over the other clones. At Tabora, survival of 60.94 and $51.56 \%$ was recorded for GC 940 and GC 584 respectively compared to the other clones. 
Table 3. Survival of 8 year old Eucalypt clones across different sites

\begin{tabular}{|c|c|c|c|c|}
\hline \multirow{2}{*}{ Treatment } & \multicolumn{4}{|c|}{ Survival (\%) } \\
\cline { 2 - 5 } & Lushoto & Kwamarukanga & Kibaha & Tabora \\
\hline GC 10 & $100.00 \mathrm{a}$ & $41.32 \mathrm{fg}$ & $34.38 \mathrm{f}$ & $40.63 \mathrm{bc}$ \\
\hline GC 14 & $99.08 \mathrm{a}$ & $42.28 \mathrm{f}$ & $50.89 \mathrm{de}$ & $43.75 \mathrm{bc}$ \\
\hline GC 15 & $100.00 \mathrm{a}$ & $93.95 \mathrm{bc}$ & $66.88 \mathrm{ab}$ & $39.06 \mathrm{bcd}$ \\
\hline GC 167 & $100.00 \mathrm{a}$ & $95.98 \mathrm{abc}$ & $54.89 \mathrm{~cd}$ & $35.94 \mathrm{bcd}$ \\
\hline GC 514 & $84.52 \mathrm{e}$ & $96.98 \mathrm{ab}$ & $31.70 \mathrm{f}$ & $42.19 \mathrm{bc}$ \\
\hline GC 581 & $98.89 \mathrm{a}$ & $89.22 \mathrm{~d}$ & $61.75 \mathrm{bc}$ & $7.81 \mathrm{~g}$ \\
\hline GC 584 & $99.05 \mathrm{a}$ & $95.13 \mathrm{abc}$ & $44.29 \mathrm{e}$ & $51.56 \mathrm{~b}$ \\
\hline GC 785 & $100.00 \mathrm{a}$ & $92.19 \mathrm{~cd}$ & $20.31 \mathrm{~g}$ & $17.19 \mathrm{fg}$ \\
\hline GC 796 & $100.00 \mathrm{a}$ & $75.23 \mathrm{~d}$ & $76.34 \mathrm{a}$ & - \\
\hline GC 940 & $93.75 \mathrm{~b}$ & $98.44 \mathrm{a}$ & $62.08 \mathrm{bc}$ & $60.94 \mathrm{a}$ \\
\hline GT 529 & $91.52 \mathrm{c}$ & $97.10 \mathrm{ab}$ & $33.52 \mathrm{f}$ & - \\
\hline GU 608 & $88.68 \mathrm{~d}$ & $37.5 \mathrm{~g}$ & & $4.69 \mathrm{gh}$ \\
\hline
\end{tabular}

Mean values in the same column with same following letters do not differ significantly ( $\mathrm{p}>0.05)$.

Table 4. Mean Dbh and height of 8 year old Eucalypt clones

\begin{tabular}{|c|c|c|c|c|c|c|c|c|}
\hline \multirow{2}{*}{ Treatment } & \multicolumn{2}{|c|}{ Lushoto } & \multicolumn{2}{c|}{ Kwamarukanga } & \multicolumn{2}{c|}{ Kibaha } & \multicolumn{2}{c|}{ Tabora } \\
\cline { 2 - 9 } & Dbh & Height & Dbh & Height & Dbh & Height & Dbh & Height \\
\hline GC 10 & $14.74 \mathrm{def}$ & $24.39 \mathrm{bcd}$ & $11.15 \mathrm{~b}$ & $21.97 \mathrm{ab}$ & $12.44 \mathrm{~g}$ & $18.36 \mathrm{bc}$ & $13.27 \mathrm{abcd}$ & $16.27 \mathrm{ab}$ \\
\hline GC 14 & $14.53 \mathrm{f}$ & $24.93 \mathrm{bcd}$ & $11.53 \mathrm{ab}$ & $22.24 \mathrm{a}$ & $13.30 \mathrm{fg}$ & $19.14 \mathrm{abc}$ & $12.56 \mathrm{abcd}$ & $16.15 \mathrm{ab}$ \\
\hline GC 15 & $15.71 \mathrm{bcd}$ & $25.94 \mathrm{bcd}$ & $12.09 \mathrm{ab}$ & $21.19 \mathrm{ab}$ & $15.59 \mathrm{abc}$ & $20.76 \mathrm{a}$ & $13.38 \mathrm{abcd}$ & $15.63 \mathrm{ab}$ \\
\hline GC 167 & $15.60 \mathrm{bcde}$ & $24.05 \mathrm{~cd}$ & $11.19 \mathrm{~b}$ & $19.18 \mathrm{bc}$ & $15.27 \mathrm{abcd}$ & $19.53 \mathrm{abc}$ & $13.21 \mathrm{abcd}$ & $14.63 \mathrm{ab}$ \\
\hline GC 514 & $15.08 \mathrm{cdef}$ & $26.36 \mathrm{bc}$ & $12.61 \mathrm{a}$ & $21.05 \mathrm{ab}$ & $15.09 \mathrm{bcde}$ & $18.94 \mathrm{abc}$ & $10.99 \mathrm{~cd}$ & $13.16 \mathrm{bc}$ \\
\hline GC 581 & $16.05 \mathrm{bc}$ & $25.90 \mathrm{bcd}$ & $11.16 \mathrm{~b}$ & $19.72 \mathrm{abc}$ & $14.23 \mathrm{cdef}$ & $18.32 \mathrm{bc}$ & $16.98 \mathrm{a}$ & $17.5 \mathrm{a}$ \\
\hline GC 584 & $16.14 \mathrm{~b}$ & $26.96 \mathrm{~b}$ & $12.18 \mathrm{ab}$ & $20.95 \mathrm{ab}$ & $14.94 \mathrm{bcdef}$ & $20.88 \mathrm{a}$ & $13.29 \mathrm{abcd}$ & $16.04 \mathrm{ab}$ \\
\hline GC 785 & $14.35 \mathrm{f}$ & $23.37 \mathrm{~d}$ & $11.01 \mathrm{bc}$ & $19.38 \mathrm{bc}$ & $13.73 \mathrm{defg}$ & $17.91 \mathrm{c}$ & $11.41 \mathrm{bcd}$ & $13.83 \mathrm{abc}$ \\
\hline GC 796 & $14.38 \mathrm{f}$ & $24.04 \mathrm{~cd}$ & $9.99 \mathrm{c}$ & $18.20 \mathrm{c}$ & $16.7 \mathrm{a}$ & $17.75 \mathrm{c}$ & - & - \\
\hline GC 940 & $14.67 \mathrm{ef}$ & $25.73 \mathrm{bcd}$ & $12.50 \mathrm{a}$ & $19.80 \mathrm{abc}$ & $14.86 \mathrm{bcdef}$ & $19.50 \mathrm{abc}$ & $13.82 \mathrm{abcd}$ & $14.5 \mathrm{abc}$ \\
\hline GT 529 & $14.46 \mathrm{f}$ & $27.05 \mathrm{~b}$ & $12.56 \mathrm{a}$ & $22.43 \mathrm{a}$ & $13.53 \mathrm{efg}$ & $19.43 \mathrm{abc}$ & - & - \\
\hline GU 608 & $19.15 \mathrm{a}$ & $30.81 \mathrm{a}$ & $12.52 \mathrm{a}$ & $20.03 \mathrm{abc}$ & $15.95 \mathrm{ab}$ & $20.33 \mathrm{ab}$ & $10.00 \mathrm{~d}$ & $10.83 \mathrm{c}$ \\
\hline
\end{tabular}

Values in the same column with same following letters do not differ significantly $(\mathrm{p}>0.05) . \quad$ Dbh $(\mathrm{cm})$ and Height $(\mathrm{m})$

\section{Diameter and Height}

The mean Dbh and height obtained from the four sites with respect to the clones studied are presented in Table 4 . GU 608, GC 584 and GC 581 had best performance in Dbh at Lushoto site while the least were GC 785, GC 796 and GT 529. GC 796, GU 608 and GC 15 outshone the other clones in Dbh at Kibaha site while GC 514, GT 529 and GU 608 outperformed GC 785, GC 796 and GC 10 at Kwamarukanga and GC 581, GU 125 and GU 21 performed relatively better than GC 514, GC 785 and GU 608 at Tabora.

As in Dbh, height of Eucalypt clones differed significant $(p<0.05)$ between clones within a site. Clone GU 608, GC 584 and GT 529 achieved significantly higher height compared to the rest of Eucalypt clones at Lushoto while GT 529, GC 14 and GC 10 had relatively higher mean height at Kwamarukanga. At Kibaha, clone GC 584, GC 15 and GU 608 showed satisfactory mean height values compared to GC 581, GC 785 and GC 796. However, GC 581, GU 125 and
GC 10 showed satisfactory values of mean height compared to GC 514, GC 796 and GU 608 for Tabora.

\section{Basal Area and Biomass Production}

Basal area and Biomass production of Eucalypt differed significant $(p<0.05)$ between clones within a site (Table 5$)$. The best clone at Lushoto site had basal area value of 28.18 $\mathrm{m}^{2} \mathrm{ha}^{-1}$ for GU 608 compared to $17.20 \mathrm{~m}^{2} \mathrm{ha}^{-1}$ for GC 514 . GT 529, GC 940 and GC 514 showed significantly higher basal area values of 12.66, 12.70 and 12.77 $\mathrm{m}^{2} \mathrm{ha}^{-1}$ respectively at Kwamarukanga site over the other clones. For Kibaha site, GC 15 and GC 940 showed significant higher basal area values of 14.1 and $14.61 \mathrm{~m}^{2} \mathrm{ha}^{-1}$ respectively over the other clones. However, at Tabora site, GC 584 and GC 940 outperformed the other clones in basal area.

The clones at Lushoto had biomass production ranging 
from 147.87 to $286.85 \mathrm{tha}^{-1}$ with the highest biomass attained by GU 608. Kwamarukanga site had biomass production ranging from 27.33 to $89.92 \mathrm{t} \mathrm{ha}^{-1}$ while at Kibaha, clones had biomass ranging 22.30 to $86.35 \mathrm{tha}^{-1}$ and 4.66 to $58.82 \mathrm{t}$ $\mathrm{ha}^{-1}$ for Tabora site (Table 5).

\section{Volume and MAI}

Volume and MAI of Eucalypts differed significantly $(p<0.05)$ between clones in all sites (Tables 6 and 7$)$. Average volume of trees at 8 years ranged from 209.23 $\mathrm{m}^{3} \mathrm{ha}^{-1}$ for GT 529 to $385.23 \mathrm{~m}^{3} \mathrm{ha}^{-1}$ for GC 608 at Lushoto.
As in Lushoto, the volume of clones at Kwamarukanga ranged from 37.81 to $124.3 \mathrm{~m}^{3} \mathrm{ha}^{-1}$ for GU 608 and GC 514 respectively. At Kibaha, GC 10 and GC 15 recorded average volume ranging between 28.07 and $103.29 \mathrm{~m}^{3} \mathrm{ha}^{-1}$ respectively while GU 21 and GC 584 recorded mean volume ranging between 7.92 and $70.88 \mathrm{~m}^{3} \mathrm{ha}^{-1}$ respectively for Tabora. As in volume, MAI was recorded ranging from 19.13 to $41.10 \mathrm{~m}^{3} \mathrm{ha}^{-1} \mathrm{yr}^{-1}$ for Lushoto, 3.41 to $21.34 \mathrm{~m}^{3} \mathrm{ha}^{-1} \mathrm{yr}^{-1}$ at Kwamarukanga (Table 6), 3.32 to 17.47 $\mathrm{m}^{3} \mathrm{ha}^{-1} \mathrm{yr}^{-1}$ for Kibaha and 0.31 to $13.76 \mathrm{~m}^{3} \mathrm{ha}^{-1} \mathrm{yr}^{-1}$ for Tabora (Table 7).

Table 5. Basal area and Biomass production of 8 year old Eucalypt clones

\begin{tabular}{|c|c|c|c|c|c|c|c|c|}
\hline \multirow{2}{*}{ Treatment } & \multicolumn{2}{|c|}{ Lushoto } & \multicolumn{2}{c|}{ Kwamarukanga } & \multicolumn{2}{c|}{ Kibaha } & \multicolumn{2}{c|}{ Tabora } \\
\cline { 2 - 10 } & Basal & Biomass & Basal & Biomass & Basal & Biomass & Basal & Biomass \\
\hline GC 10 & $19.21 \mathrm{e}$ & $170.56 \mathrm{~d}$ & $3.97 \mathrm{~g}$ & $27.45 \mathrm{~g}$ & $4.16 \mathrm{e}$ & $22.30 \mathrm{e}$ & $5.67 \mathrm{~cd}$ & $32.07 \mathrm{bc}$ \\
\hline GC 14 & $18.29 \mathrm{f}$ & $159.23 \mathrm{efg}$ & $4.62 \mathrm{f}$ & $32.12 \mathrm{f}$ & $4.16 \mathrm{e}$ & $23.16 \mathrm{e}$ & $6.13 \mathrm{~cd}$ & $34.88 \mathrm{bc}$ \\
\hline GC 15 & $21.02 \mathrm{~d}$ & $186.58 \mathrm{c}$ & $11.69 \mathrm{~b}$ & $82.09 \mathrm{~b}$ & $14.61 \mathrm{a}$ & $86.35 \mathrm{a}$ & $6.64 \mathrm{~cd}$ & $37.67 \mathrm{bc}$ \\
\hline GC 167 & $21.24 \mathrm{~cd}$ & $192.75 \mathrm{~b}$ & $10.14 \mathrm{c}$ & $70.59 \mathrm{c}$ & $11.44 \mathrm{~b}$ & $67.74 \mathrm{~b}$ & $5.84 \mathrm{~cd}$ & $33.09 \mathrm{bc}$ \\
\hline GC 514 & $17.20 \mathrm{~g}$ & $153.60 \mathrm{gh}$ & $12.77 \mathrm{a}$ & $89.76 \mathrm{a}$ & $10.69 \mathrm{~b}$ & $61.94 \mathrm{~b}$ & $4.61 \mathrm{de}$ & $26.74 \mathrm{~cd}$ \\
\hline GC 581 & $21.76 \mathrm{bc}$ & $196.29 \mathrm{~b}$ & $9.34 \mathrm{~d}$ & $64.95 \mathrm{~d}$ & $5.04 \mathrm{e}$ & $28.70 \mathrm{e}$ & $2.78 \mathrm{ef}$ & $14.82 \mathrm{ef}$ \\
\hline GC 584 & $21.94 \mathrm{~b}$ & $197.77 \mathrm{~b}$ & $11.74 \mathrm{~b}$ & $82.20 \mathrm{~b}$ & $11.16 \mathrm{~b}$ & $65.22 \mathrm{~b}$ & $10.00 \mathrm{ab}$ & $57.16 \mathrm{a}$ \\
\hline GC 785 & $18.07 \mathrm{f}$ & $156.84 \mathrm{fg}$ & $9.54 \mathrm{~d}$ & $66.35 \mathrm{~d}$ & $6.42 \mathrm{~d}$ & $36.01 \mathrm{~d}$ & $3.09 \mathrm{ef}$ & $18.13 \mathrm{de}$ \\
\hline GC 796 & $18.49 \mathrm{f}$ & $163.92 \mathrm{e}$ & $6.53 \mathrm{e}$ & $45.11 \mathrm{e}$ & $4.18 \mathrm{e}$ & $25.98 \mathrm{e}$ & - & - \\
\hline GC 940 & $18.06 \mathrm{f}$ & $160.64 \mathrm{ef}$ & $12.70 \mathrm{a}$ & $89.07 \mathrm{a}$ & $14.61 \mathrm{a}$ & $84.12 \mathrm{a}$ & $10.51 \mathrm{a}$ & $58.82 \mathrm{a}$ \\
\hline GT 529 & $16.88 \mathrm{gf}$ & $147.87 \mathrm{~h}$ & $12.66 \mathrm{a}$ & $89.92 \mathrm{a}$ & $9.54 \mathrm{c}$ & $52.57 \mathrm{c}$ & - & - \\
\hline GU 608 & $28.18 \mathrm{a}$ & $286.85 \mathrm{a}$ & $3.88 \mathrm{~g}$ & $27.33 \mathrm{~g}$ & $6.67 \mathrm{~d}$ & $39.35 \mathrm{~d}$ & $0.78 \mathrm{f}$ & $4.66 \mathrm{f}$ \\
\hline
\end{tabular}

Values in the same column with same following letters do not differ significantly $(\mathrm{p}>0.05)$. Basal area $\left(\mathrm{m}^{2} \mathrm{ha}^{-1}\right)$ and Biomass $\left(\mathrm{t}\right.$ ha $\left.{ }^{-1}\right)$

Table 6. Volume and MAI of 8 year old Eucalypt clones

\begin{tabular}{|c|c|c|c|c|c|c|c|c|c|c|}
\hline \multirow{3}{*}{ Treatment } & \multirow{2}{*}{\multicolumn{4}{|c|}{$\begin{array}{c}\text { Volume }\left(\mathrm{m}^{3} \mathrm{ha}^{-1}\right) \\
\text { Lushoto }\end{array}$}} & \multirow{3}{*}{ MAI } & \multirow{2}{*}{\multicolumn{4}{|c|}{$\begin{array}{l}\text { Volume }\left(m^{3} h^{-1}\right) \\
\text { Kwamarukanga }\end{array}$}} & \multirow{3}{*}{ MAI } \\
\hline & & & & & & & & & & \\
\hline & 2007 & 2008 & 2009 & 2012 & & 2007 & 2008 & 2009 & 2012 & \\
\hline GC 10 & $116.14 f$ & $160.31 \mathrm{e}$ & $161.81 \mathrm{f}$ & $239.99 d$ & $25.65 \mathrm{~d}$ & 60.96 & $60.11 \mathrm{f}$ & $61.30 \mathrm{f}$ & $38.22 \mathrm{~g}$ & $7.59 \mathrm{~g}$ \\
\hline GC 14 & $111.75 \mathrm{~g}$ & $155.30 \mathrm{f}$ & $166.91 \mathrm{e}$ & $225.67 \mathrm{e}$ & $26.2 \mathrm{~cd}$ & $53.55 \mathrm{f}$ & $62.70 \mathrm{e}$ & $60.47 \mathrm{f}$ & $44.64 \mathrm{f}$ & $7.92 \mathrm{~g}$ \\
\hline GC 15 & $134.19 \mathrm{~d}$ & $182.14 \mathrm{c}$ & $182.94 \mathrm{c}$ & $262.88 \mathrm{c}$ & $26.91 \mathrm{c}$ & $75.36 \mathrm{a}$ & $84.44 \mathrm{~b}$ & $98.92 b$ & $113.7 \mathrm{~b}$ & $19.1 \mathrm{bc}$ \\
\hline GC 167 & $139.82 b$ & $193.5 \mathrm{~b}$ & $199.54 a$ & $269.3 \mathrm{bc}$ & $29.20 \mathrm{~b}$ & $67.14 \mathrm{c}$ & $69.59 \mathrm{~d}$ & $81.95 \mathrm{~d}$ & $98.05 \mathrm{c}$ & $15.0 \mathrm{~d}$ \\
\hline GC 514 & $111.33 \mathrm{~g}$ & $140.8 \mathrm{~g}$ & $140.77 \mathrm{i}$ & $215.73 \mathrm{fg}$ & $19.81 \mathrm{~g}$ & $67.8 \mathrm{bc}$ & $82.37 \mathrm{~b}$ & $97.78 \mathrm{~b}$ & $124.3 \mathrm{a}$ & $19.5 \mathrm{~b}$ \\
\hline GC 581 & $139.46 \mathrm{~b}$ & $198.57 \mathrm{a}$ & $198.96 a$ & $274.94 \mathrm{~b}$ & $29.89 \mathrm{~b}$ & $61.92 \mathrm{~d}$ & $70.78 \mathrm{~d}$ & $81.51 \mathrm{~d}$ & $90.26 \mathrm{~d}$ & $14.22 \mathrm{e}$ \\
\hline GC 584 & $137.7 \mathrm{bc}$ & $193.6 \mathrm{~b}$ & $194.13 b$ & $277.16 b$ & $29.78 b$ & $63.69 \mathrm{~d}$ & $74.67 \mathrm{c}$ & $92.79 \mathrm{c}$ & $114.3 \mathrm{~b}$ & $18.61 \mathrm{c}$ \\
\hline GC 785 & $122.35 \mathrm{e}$ & $175.2 \mathrm{~d}$ & $173.81 \mathrm{~d}$ & $222.40 \mathrm{ef}$ & $24.11 \mathrm{e}$ & $57.96 \mathrm{e}$ & $63.65 \mathrm{e}$ & $76.35 \mathrm{e}$ & $92.21 \mathrm{~d}$ & $13.72 \mathrm{e}$ \\
\hline GC 796 & $135.4 \mathrm{~cd}$ & $182.37 \mathrm{c}$ & $185.99 \mathrm{c}$ & $230.60 \mathrm{e}$ & $22.07 \mathrm{f}$ & $48.26 \mathrm{~g}$ & $52.01 \mathrm{~g}$ & $58.77 \mathrm{f}$ & $62.83 \mathrm{e}$ & $9.33 \mathrm{f}$ \\
\hline GC 940 & $120.44 \mathrm{e}$ & $156.18 \mathrm{f}$ & $156.22 \mathrm{~g}$ & $225.86 \mathrm{e}$ & $21.21 \mathrm{f}$ & $70.38 \mathrm{~b}$ & $83.76 \mathrm{~b}$ & $98.51 \mathrm{~b}$ & $123.4 \mathrm{a}$ & $19.7 \mathrm{~b}$ \\
\hline GT 529 & $113.66 \mathrm{fg}$ & $143.6 \mathrm{~g}$ & $145.73 \mathrm{~h}$ & $209.23 \mathrm{~g}$ & $19.13 \mathrm{~g}$ & $75.09 \mathrm{a}$ & $87.54 \mathrm{a}$ & $114 \mathrm{a}$ & $123.2 \mathrm{a}$ & $21.34 \mathrm{a}$ \\
\hline GU 608 & $150.83 \mathrm{a}$ & $198.21 \mathrm{a}$ & $200.06 a$ & $385.23 \mathrm{a}$ & $41.10 \mathrm{a}$ & $31.28 \mathrm{~h}$ & $25.47 \mathrm{~h}$ & $31.61 \mathrm{~g}$ & $37.81 \mathrm{~g}$ & $3.41 \mathrm{~h}$ \\
\hline
\end{tabular}

Note: MAI in $\left(\mathrm{m}^{3} \mathrm{ha}^{-1} \mathrm{y}^{-1}\right)$, Mean values in the same column with same following letters do not differ significantly $(\mathrm{p}>0.05)$. 
Table7. Volume and MAI of 8 year old Eucalypt clones

\begin{tabular}{|c|c|c|c|c|c|c|c|c|c|}
\hline \multirow{2}{*}{ Treatment } & Kibaha & & & & \multirow{2}{*}{ MAI } & & \multirow{2}{*}{$\begin{array}{c}\text { Tabora } \\
2008 \\
\end{array}$} & \multirow{2}{*}{2012} & \multirow{2}{*}{ MAI } \\
\hline & 2007 & 2008 & 2009 & 2012 & & 2007 & & & \\
\hline GC 10 & $38.84 d$ & $38.08 \mathrm{e}$ & $38.56 \mathrm{e}$ & $28.07 \mathrm{e}$ & $4.18 \mathrm{f}$ & $14.10 \mathrm{~cd}$ & $16.70 \mathrm{f}$ & $39.88 \mathrm{bc}$ & $6.31 \mathrm{ab}$ \\
\hline GC 14 & 34.664 & $27.82 \mathrm{f}$ & $25.2 \mathrm{~g}$ & $28.57 \mathrm{e}$ & $3.32 \mathrm{f}$ & $12.12 \mathrm{~d}$ & $27.76 \mathrm{~cd}$ & $43.29 b c$ & $9.95 \mathrm{ab}$ \\
\hline GC 15 & $58.06 \mathrm{a}$ & $71.44 \mathrm{a}$ & $84.36 \mathrm{a}$ & $103.29 \mathrm{a}$ & $17.47 \mathrm{a}$ & $25.71 \mathrm{a}$ & $43.54 \mathrm{a}$ & $46.82 b c$ & $8.77 \mathrm{ab}$ \\
\hline GC 167 & $46.9 \mathrm{c}$ & $57.46 \mathrm{c}$ & $65.28 \mathrm{~b}$ & $80.92 \mathrm{~b}$ & $13.22 \mathrm{c}$ & $22.18 \mathrm{a}$ & $35.01 \mathrm{~b}$ & $41.13 b c$ & $6.81 \mathrm{ab}$ \\
\hline GC 514 & $46.71 \mathrm{c}$ & $57.08 \mathrm{c}$ & $58.74 \mathrm{c}$ & $74.87 \mathrm{~b}$ & $12.14 \mathrm{c}$ & $13.52 \mathrm{~cd}$ & $21.84 \mathrm{def}$ & $33.01 \mathrm{~cd}$ & $5.67 \mathrm{ab}$ \\
\hline GC 581 & $34.70 \mathrm{~d}$ & $40.70 \mathrm{de}$ & $42.55 \mathrm{e}$ & $34.99 \mathrm{e}$ & $6.04 \mathrm{e}$ & $23.23 \mathrm{a}$ & $40.75 \mathrm{a}$ & $18.78 \mathrm{def}$ & $8.14 \mathrm{ab}$ \\
\hline GC 584 & $55.37 \mathrm{ab}$ & $61.86 \mathrm{~b}$ & $68.78 \mathrm{~b}$ & $78.47 \mathrm{~b}$ & $13.19 \mathrm{c}$ & $14.74 \mathrm{bcd}$ & $25.86 \mathrm{~cd}$ & $70.88 \mathrm{a}$ & $10.98 \mathrm{ab}$ \\
\hline GC 785 & $39.12 d$ & $43.23 \mathrm{~d}$ & $41.73 \mathrm{e}$ & $44.27 \mathrm{~d}$ & $6.12 \mathrm{e}$ & $12.25 \mathrm{~d}$ & $19.98 \mathrm{fe}$ & $22.30 \mathrm{de}$ & $5.66 \mathrm{ab}$ \\
\hline GC 796 & $32.12 \mathrm{e}$ & $21.57 \mathrm{~g}$ & $26.28 \mathrm{~g}$ & $30.25 \mathrm{e}$ & $5.34 \mathrm{e}$ & - & - & - & - \\
\hline GC 940 & $52.37 \mathrm{~b}$ & $71.62 \mathrm{a}$ & $81.09 \mathrm{a}$ & $102.01 \mathrm{a}$ & $15.73 b$ & $25.51 \mathrm{a}$ & $45.08 \mathrm{a}$ & $73.42 \mathrm{a}$ & $13.76 \mathrm{a}$ \\
\hline GT 529 & $45.87 \mathrm{c}$ & $54.12 \mathrm{c}$ & $50.92 \mathrm{~d}$ & $65.24 \mathrm{c}$ & $9.02 \mathrm{~d}$ & - & - & - & - \\
\hline GU 608 & $35.42 \mathrm{de}$ & $28.87 \mathrm{f}$ & $32.73 \mathrm{f}$ & $45.12 d$ & $6.25 \mathrm{e}$ & $13.87 \mathrm{~cd}$ & $16.44 \mathrm{f}$ & $5.69 \mathrm{f}$ & $0.31 \mathrm{~b}$ \\
\hline
\end{tabular}

Note: MAI in $\left(\mathrm{m}^{3} \mathrm{ha}^{-1} \mathrm{y}^{-1}\right)$, Mean values in the same column with same following letters do not differ significantly $(\mathrm{p}>0.05)$.

\section{Ordinal Ranking}

The overall best performing clones for each site were ranked. GC 581, GC 584 and GU 608 showed better performance for Lushoto while GC 15, GC 167 and GC 940 showed better growth performance at Kibaha. GC 514, GT 529 and GC 940 performed better at Kwamarukanga while GU 15, GC 584 and GC 940 scored higher than other clones at Tabora site. Ordinal ranking values reported in this study show that, GC 940 grows well in different climatic conditions. This indicates that rainfall, temperature, soil and altitude of the study sites are within the optimal range for the survival and growth of GC 940 .

\section{Discussion}

Eucalypt clones studies have yielded mixed results both in terms of survival, growth performance and productivity. The survival of most of clones at Lushoto and Kwamarukanga were more than $95 \%$. These finding are similar to those reported by [15-16] that the survival percentage of the majority of clonal plantations is more than $95 \%$. Moreover, the difference in survival between clones within a site observed was probably a result of genetic differences between the clones which interact differently with the various climatic and soil conditions. The varied survival trends between clones within a site showed a strong environment clone interaction, an observation supported by $[10,17]$. For Tabora site, low survival was probably due to fire outbreak which occurred in 2009 and other human disturbances. Survival in general is influenced by several factors, which include site management, especially the weeding frequency and the protection of the seedlings from pests and diseases, drought and seedling handling during planting period [18].

With respect to Dbh, Eucalypt clones studied showed similar Dbh trends as reported by various researchers. For example, [19-21] in Congo, Kenya and North Iraq reported that 5 to 8 year old Eucalypt clones had a mean Dbh of 14 to $16 \mathrm{~cm}$ while [22] reported mean Dbh of $13 \mathrm{~cm}$ of 8 year old Eucalyptus nitens in coastal Ireland. Similarly, significant differences in Eucalypt clones have been reported by various researchers. For example, [10, 23] observed significant differences for growth attributes among seven species of Eucalypt clones and found that, Indian clones had higher promising performance for Dbh than the results of this study. These differences in Dbh between clones within a site may be attributed to genetic difference [17].

On the other hand, mean height of GU 608 at Lushoto was significantly higher than $28 \mathrm{~m}$ for a 7 year old Eucalypt clonal plantation in Congo [19], 11 to $14 \mathrm{~m}$ for 8 year old Eucalyptus nitens [22] and $12.5 \mathrm{~m}$ and $19.9 \mathrm{~m}$ for Eucalypt clones in Kenya [18]. The performance of Eucalypt clones studied encouraged the expansion of plantations and woodlot forests of Eucalypts to reducing pressure to the few remaining natural forests in Tanzania. However, height growth, especially in Eucalypts which are shade intolerant is strongly affected by stocking i.e. number of stems per unit area [24]. High height growth was observed between clones at Lushoto and Kwamarukanga sites due to high survival rate. However, clones at Kibaha and Tabora sites due to low survival rate resulting into wider spacing with no competition for light, the height growth between clones was observed to be low.

In addition, [26] reported that 15 year old E. resinifera had similar basal area to Lushoto site but higher than results from Kwamarukanga, Kibaha and Tabora. Reference [19] Reported lower results compared to clones at Lushoto highland area but higher to the other studied sites. The study results are lower than those reported by [25] for various Eucalypt clones in Brazil. Significant difference observed in basal area between clones within a site suggests that their 
level of adaptation to site conditions is different. GC clones are more suited for growing in medium agricultural potential areas receiving annual rainfall above $750 \mathrm{~mm}$ and at an elevation of less than 1700 m.a.s.l [27]. The GCs are not suitable for growing in semi-arid areas where $E$. camaldulensis does best or above 1700 m.a.s.l. where $E$. grandis grows best. In high rainfall areas with amounts over $1200 \mathrm{~mm}$ a year, the growth rate of GCs is lower than that of widely grown local E. grandis [17].

The clones at Lushoto, in particular, outshone the other sites in terms of having high biomass production ranging from 147.87 to $286.85 \mathrm{t} \mathrm{ha}^{-1}$ with the highest biomass attained by GU 608. Growth differences have been found in many cold tolerant Eucalypt species tested under South African growing conditions [28-29], signifying the importance of site-species matching, as well as site-provenance matching [30-31]. Differences between genetic materials of Eucalypts as to biomass yield and distribution have been attributed to varying adaptability to local conditions as reported by several authors in [32]. Biomass accumulation is a result of greater or more effective capture of growth inducing resources such as water, nutrients and/or solar radiation. Findings from this study compare well with several data presented by [33-34] including biomass production per year of some fast growing Eucalyptus species shows clearly the strong potential of Eucalypts for wood production. According to [35] total biomass differed between clones and among $E$. grandis x E. urophylla (GU) clones. The findings of this study are similar to those of [19, 36].

Moreover, the productivity of Eucalypt clones falls in the range reported for some Eucalypt hybrid plantations $(E$. urophylla $\times E$. grandis) in South Africa, South America, India, Brazil and Cameron [37-39]. The superior volume for clones at Lushoto site might be attributed to sites advantages in good growth in diameter and height ascribed to the favourable climatic conditions, especially the high rainfall and soil type. This implies that Eucalypt clones are better adapted to altitudes ranging from 1393-1486 m.a.s.l with rainfall above $1000 \mathrm{~mm}$ and temperature ranging between $7^{\circ} \mathrm{C}$ and $30^{\circ} \mathrm{C}$.

The growth of trees is mainly influenced by different factors including genotype, environment and management. It was evident that clones at Lushoto performed better, showing the influence of environment on tree growth. The differences in performance among Eucalypt clones at various sites indicate that some clones are more adaptable to specific sites 17, 40]. According to [10] different sites have different level of fertility, soil texture etc. GU 608 performed well at Lushoto site only, implying that they are not suitable in low land and dry areas. Similar observations have been made by [41] that GUs was not suitable for lowland humid tropics.

\section{Conclusions}

Eucalypt clones studied showed significant differences in terms of survival, Dbh, height, basal area, volume and biomass production within a site with the greatest growth performance being shown at Lushoto. The productivity of all clones in Lushoto site is better than those obtained elsewhere in the world. It is recommended that, GC 581, GC 584 and GU 608 are better for Lushoto. GC 15, GC 167 and GC 940 better for Kibaha. GC 514, GT 529 and GC 940 recommended for Kwamarukanga and GC 15, GC 584 and GC 940 recommended for Tabora. They are also recommended for planting in areas with climatic and soil conditions similar to the sites where they were tested.

\section{Acknowledgements}

We thank the Commission for Science and Technology financing this study. The authors are also grateful to TAFORI for permitting use of trials.

\section{REFERENCES}

[1] J.D. Nichols, R.G.B Smith, J.C Grant, K. Glencross. 2010. Subtropical eucalypt plantations in Eastern Australia. Journal of Australian Forest 73: 53 - 62 .

[2] H. Zegeye. 2010. Environmental and socio-economic implications of Eucalyptus in Ethiopia. In: Gil, L., Wubalem Tadesse, Tolosana, E. and López, R. (eds.), Proceedings of the Conference on Eucalyptus Species Management, History, Status and Trends in Ethiopian Institute of Agricultural Research, Addis Ababa. pp. 184 - 205.

[3] P.K.T. Munishi. 2007. The Eucalyptus controversies in Tanzania. TAF Annual General meeting, Dodoma, Tanzania.

[4] Y. Ngaga. 2011. Forest plantations and woodlots in Tanzania. African Forest Forum (16): 1 - 80, 2011.

[5] C.D. Whitesell, D.S. DeBell, T.H. Schubert, R.F. Strand, T.B. Crabb. 1992. Short-rotation Management of Eucalyptus: Guidelines for Plantations in Hawaii. Forest Service General Technical Report No.137. United States Department of Agriculture, USA. 30pp.

[6] F. Mwaniki, G. Muluvi, C. Gichuki, V.O. Oeba, B. Kanyi. 2009. Development of non-mist vegetative propagation protocol for Eucalyptus Hybrid Clones. JEANRM 3(1): 283 295.

[7] J.P. Chandra, M.P.S. Yadava. 1986. Clonal propagation of Mysore gum (Eucalyptus hybrid). Indian Forester 112: 783 $791,1986$.

[8] P. Mwangingo, T.H. Msangi. 2004. Towards optimizing the benefits of clonal forestry to small-scale farmers in East Africa. Workshop Report of the International Service for the Acquisition of Agri-Biotech Applications Brief. (Edited by Ngamau, C., Kanyi, B., Epila-Otara, J., Mwangingo, P. and Wakhusama, S.), 26-27 January 2004, Nairobi, Kenya. pp. 17 -20 .

[9] P. Oballa, E. Chagala-Odera, L. Wamalwa, V. Oeba, E. 
Mutitu, L. Mwangi. 2005. The Performance of Eucalyptus Hybrid Clones and Local Landraces in Various Agro-Ecological Zones in Kenya. International Service for the Acquisition of Agri-Biotechnology Applications, Nairobi, Kenya. $121 \mathrm{pp}$.

[10] I.D. Arya, S. Sharma, S. Arya. 2009. Micropropagation of superior eucalyptus hybrids FRI-5 (E. camaldulensis Dehn x E. tereticornis Sm) and FRI-14 (E. torelliana F.V. Muell x E. citriodora Hook): A commercial multiplication and field evaluation. African Journal of Biotechnology 8(21): $5718-5726$

[11] T.H. Msangi, C.F. Shangali, W. Mugasha, J. Maguzu, F.A. Bomani. 2009. Early growth of various Eucalyptus hybrid clones in different agro-ecosystems in Tanzania. JEANRM 3(1): $249-251$.

[12] P.W. West. 2004. Tree and Forest Measurement. Germany Springer, Berlín. 167pp.

[13] D. Zianis, P. Muukkonen, R. Mäkipää, M. Mencuccini. Biomass and stem volume equations for tree species in Europe. Silva Fennica Monographs 4: 1 - 63, 2005.

[14] W.A. Mugasha, T. Eid, O.M. Bollandsås, R.E. Malimbwi, S.A.O. Chamshama, E. Zahabu, J.Z. Katani. 2013. Allometric models for prediction of above- and belowground biomassof trees in the miombo woodlands of Tanzania. Forest Ecology and Management 310: 87-101.

[15] H.D. Kulkarni, P. Lal. 1995. Performance of eucalyptus clones at ITC Bhadrachalam India. In: Potts, B. M., et. al., (Ed.). Eucalyptus plantations: improving fibre yield and quality. Proc. CRCTHF - IUFRO Cong., Hobart, Australia. pp. $274-275$.

[16] M.A. Drumond, V.R. de Oliveira, J.A. Tavares, J. Ribaski, P.E.T. dos Santos. 2012. Performance of two hybrid clones of Eucalyptus planted under five spacings in the Araripe plateau, Pernambuco, Brazil. International Society for Horticultural Science 959: 167 - 172.

[17] L. Wamalwa, E. Chagala-Odera, V. Oeba, P.O Oballa. 2007. Adaptability of four-year old Eucalyptus species and clones in Kenya. Discovery and Innovation 19(4): 326 - 334.

[18] S.W. Kahunyo. 2008. Evaluation of growth performance of eucalyptus tree hybrids planted in various agroclimatic areas in Kenya. Dissertation for Award of MSc Degree at Kenyatta University, Nairobi, Kenya, 198pp.

[19] F. Bernhard-Reversat (Ed). 2001. Effect of exotic tree plantations on plant diversity and biological soil fertility in the Congo savannah with special reference to Eucalypts. CIFOR, Bogor, Indonesia. 71pp.

[20] B.B. Kirongo, G.K. Kimani, M. Muchiri. 2008. Five year growth and survival of Eucalyptus hybrid clones in Coastal Kenya. Proceedings of 4th Annual International Conference, Moi University July 29 - Aug 1, 2008. pp. 21 - 30.

[21] N.A. Qader, N.A. Ab-Shukor, A.S.M. Roseley. 2014. Selection of plus tree based on growth performance and fibre morphology characteristics as improved sources for propagation of Eucalyptus camaldulensis. American Journal of Plant Sciences 5: 1329 - 1335.

[22] J. Neilan, D. Thompson. 2008. Eucalyptus as a potential biomass species for Ireland.
[23] R. Kumar, K.S. Bangarwa. 2006. Clonal evaluation in Eucalyptus tereticornis Sm. Environmental Ecology 24(4): $1188-1191$

[24] E. Varis. 2011. Stand growth and management scenarios for Paraserianthes falcataria smallholder plantations in Indonesia. Thesis for Award of MSc Degree in Forest Ecology at University of Helsinki, Indonesia, 102pp.

[25] L.M.B. Rossi, C.P. Azevedo, C.R. Souza, R.M.B. Lima. 2003. Potential forest species for plantations in Brazilian Amazonia. Forestry 23: 238-247.

[26] C. Delgado-Matas, T. Pukkala. 2011. Comparison of the Growth of Six Eucalyptus Species in Angola. International Journal of Forestry Research 2011: 1 -9.

[27] P.O. Oballa, P.K.A. Konuche, M.N. Muchiri, B.N. Kigomo. 2009. Facts on Growing and Use of Eucalyptus in Kenya. Kenya Forestry Research Institute, Nairobi, Kenya. 37pp.

[28] T.L. Swain, C.T. Chiappero, R.A.W. Gardner. 1998. Final Measurements of Six Nitens provenance/Progeny Trials in the Summer Rainfall Region of South Africa. ICFR Bulletin Series No. 5. ICFR, Pietermaritzburg.

[29] T.L. Swain, R.A.W. Gardner. 2000. Eucalyptus dunnii provenance/progeny trials in KwaZulu-Natal, South Africa final measurements and pulping properties. In: Proceedings of IUFRO Working Group 2.08.01 Conference of Forest Genetics for the Next Millennium. Durban, October 2000.

[30] T.L. Swain, R.A.W. Gardner. 2002. Use of site-species matching and genetic gain to maximise yield - a South African example. In: Proceedings International Symposium on Eucalyptus Plantations. Guangzhou Zhaoqing, Guandong, China, 1 - 6 September 2002.

[31] T.L. Swain, R.A.W. Gardner. 2003. A Summary of Current Knowledge of Cold Tolerant Eucalypt Species Grown in South Africa. Bulletin Series No. 3. Institute for Commercial Forestry Research, Pietermaritzburg.

[32] T.C.G.R. Andrade, N.F. de Barros, L.E. Dias, M.I.R. Azevedo. Biomass yield and calolific value of six clonal stands of Eucalyptus urophylla S T. Blake cultivated in northeastern Brazil. Cerne Lavras 19(3): 467 - 472.

[33] E.A. Hansen, J.B. Baker. 1979. Biomass and Nutrient Removal in Short Rotation Intensively Cultured Plantations. Annual meeting North American Poplars Council, Thompsonville. $21 \mathrm{pp}$.

[34] F. Poggiani, H.T.Z. Couto. 1983. Biomass and nutrient estimates in short rotation intensively cultured plantation of Eucalyptus grandis. IPEF Presented at the IUFRO Fast-Growing Trees Symposium, Aguas de SãoPedro - S. Paulo, August 1980. pp.29-36.

[35] R. Safou-Matondo, P. Deleporte, J.P. Laclau, J.P. Bouillet. 2005. Hybrid and clonal variability of nutrient content and nutrient use efficiency in Eucalyptus stands in Congo. Forest Ecology and Management 210(3): 193 -204.

[36] J.L.M. Gonçalves, N.F. De Barros. 1999. Improvement of site productivity for short-rotation plantations in Brazil. Bosque 20(1): $89-106$.

[37] R.K. Luna, N.S. Thakur, V. Kumar. 2009. Performance of clonal Eucalyptus in different agro-climatic zones of Punjab. Indian Forester 135(5): 1455 - 1464. 
[38] A.C. Almeida, A. Siggins, T.R. Batista, C. Beadle, S. Fonseca, R, Loos. 2010. Mapping the effect of spatial and temporal variation in climate and soils on Eucalyptus plantation production with 3-PG, a process-based growth model. Forest Ecology and Management 259:1730 - 1740.

[39] R. Pérez-Sandoval, A. Gómez-Guerrero A. Fierros-González, W.R. Horwath. 2012. Site productivity of clone and seed raised plantations of Eucalyptus urophylla and Eucalyptus grandis in Southeast Mexico. Open Journal of Forestry 2(4): $225-231$
[40] B.B. Kirongo, M.N. Muchiri. 2009. Modeling early growth of Eucalyptus hybrid clones at the Kenyan coast. Journal of East African Natural Resources Management 3: 257-271.

[41] E.B. Hardiyanto. 1996. The status of genetic resources utilization for industrial forest plantation in Indonesia. Proceeding International Seminar on Tropical Plantation Establishment-Improving Productivity Through Genetic Practices. Yogyakarta, Indonesia, 19 - 21 December 1996. $120 \mathrm{pp}$. 